\title{
On Adversarial Removal of Hypothesis-only Bias in Natural Language Inference
}

\author{
Yonatan Belinkov $^{13 *} \quad$ Adam Poliak $^{2 *}$ \\ Stuart M. Shieber ${ }^{1} \quad$ Benjamin Van Durme $^{2} \quad$ Alexander Rush $^{1}$ \\ ${ }^{1}$ Harvard University $\quad{ }^{2}$ Johns Hopkins University $\quad{ }^{3}$ Massachusetts Institute of Technology \\ \{belinkov, shieber, srush\}@seas.harvard.edu \\ \{azpoliak, vandurme\}@cs.jhu.edu
}

\begin{abstract}
Popular Natural Language Inference (NLI) datasets have been shown to be tainted by hypothesis-only biases. Adversarial learning may help models ignore sensitive biases and spurious correlations in data. We evaluate whether adversarial learning can be used in NLI to encourage models to learn representations free of hypothesis-only biases. Our analyses indicate that the representations learned via adversarial learning may be less biased, with only small drops in NLI accuracy.
\end{abstract}

\section{Introduction}

Popular datasets for Natural Language Inference (NLI) - the task of determining whether one sentence (premise) likely entails another (hypothesis) - contain hypothesis-only biases that allow models to perform the task surprisingly well by only considering hypotheses while ignoring the corresponding premises. For instance, such a method correctly predicted the examples in Table 1 as contradictions. As datasets may always contain biases, it is important to analyze whether, and to what extent, models are immune to or rely on known biases. Furthermore, it is important to build models that can overcome these biases.

Recent work in NLP aims to build more robust systems using adversarial methods (Alzantot et al., 2018; Chen \& Cardie, 2018; Belinkov \& Bisk, 2018, i.a.). In particular, Elazar \& Goldberg (2018) attempted to use adversarial training to remove demographic attributes from text data, with limited success. Inspired by this line of work, we use adversarial learning to add small components to an existing and popular NLI system that has been used to learn general sentence representations (Conneau et al., 2017). The adversarial

\footnotetext{
* Equal contribution
}

A dog runs through the woods near a cottage

- The dog is sleeping on the ground

A person writing something on a newspaper

- A person is driving a fire truck

A man is doing tricks on a skateboard

Nobody is doing tricks

Table 1: Examples from SNLI's development set that Poliak et al. (2018)'s hypothesis-only model correctly predicted as contradictions. The first line in each section is a premise and lines with - are corresponding hypotheses. The italicized words are correlated with the "contradiction" label in SNLI

techniques include (1) using an external adversarial classifier conditioned on hypotheses alone, and (2) creating noisy, perturbed training examples. In our analyses we ask whether hidden, hypothesisonly biases are no longer present in the resulting sentence representations after adversarial learning. The goal is to build models with less bias, ideally while limiting the inevitable degradation in task performance. Our results suggest that progress on this goal may depend on which adversarial learning techniques are used.

Although recent work has applied adversarial learning to NLI (Minervini \& Riedel, 2018; Kang et al., 2018), this is the first work to our knowledge that explicitly studies NLI models designed to ignore hypothesis-only biases.

\section{Methods}

We consider two types of adversarial methods. In the first method, we incorporate an external classifier to force the hypothesis-encoder to ignore hypothesis-only biases. In the second method, we randomly swap premises in the training set to create noisy examples. 


\subsection{General NLI Model}

Let $(P, H)$ denote a premise-hypothesis pair, $g$ denote an encoder that maps a sentence $S$ to a vector representation $\boldsymbol{v}$, and $c$ a classifier that maps $\boldsymbol{v}$ to an output label $y$. A general NLI framework contains the following components:

- A premise encoder $g_{P}$ that maps the premise $P$ to a vector representation $\boldsymbol{p}$.

- A hypothesis encoder $g_{H}$ that maps the hypothesis $H$ to a vector representation $\boldsymbol{h}$.

- A classifier $c_{\mathrm{NLI}}$ that combines and maps $\boldsymbol{p}$ and $\boldsymbol{h}$ to an output $y$.

In this model, the premise and hypothesis are each encoded with separate encoders. The NLI classifier is usually trained to minimize the objective:

$$
L_{\mathrm{NLI}}=L\left(c_{\mathrm{NLI}}\left(\left[g_{P}(P) ; g_{H}(H)\right], y\right)\right)
$$

where $L(\tilde{y}, y)$ is the cross-entropy loss. If $g_{P}$ is not used, a model should not be able to successfully perform NLI. However, models without $g_{P}$ may achieve non-trivial results, indicating the existence of biases in hypotheses (Gururangan et al., 2018; Poliak et al., 2018; Tsuchiya, 2018).

\subsection{AdvCls: Adversarial Classifier}

Our first approach, referred to as AdvCls, follows the common adversarial training method (Goodfellow et al., 2015; Ganin \& Lempitsky, 2015; Xie et al., 2017; Zhang et al., 2018) by adding an additional adversarial classifier $c_{\text {Hypoth }}$ to our model. $c_{\text {Hypoth }}$ maps the hypothesis representation $\boldsymbol{h}$ to an output $y$. In domain adversarial learning, the classifier is typically used to predict unwanted features, e.g., protected attributes like race, age, or gender (Elazar \& Goldberg, 2018). Here, we do not have explicit protected attributes but rather latent hypothesis-only biases. Therefore, we use $c_{\text {Hypoth }}$ to predict the NLI label given only the hypothesis. To successfully perform this prediction, $c_{\text {Hypoth }}$ needs to exploit latent biases in $\boldsymbol{h}$.

We modify the objective function (1) as

$$
\begin{aligned}
& L=L_{\mathrm{NLI}}+\lambda_{\mathrm{Loss}} L_{\mathrm{Adv}}
\end{aligned}
$$

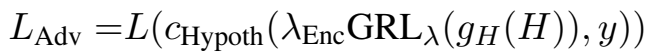

To control the interplay between $c_{\mathrm{NLI}}$ and $c_{\mathrm{Hypoth}}$ we set two hyper-parameters: $\lambda_{\text {Loss }}$, the importance of the adversarial loss function, and $\lambda_{\mathrm{Enc}}$, a scaling factor that multiplies the gradients after reversing them. This is implemented by the scaled gradient reversal layer, $\mathrm{GRL}_{\lambda}$ (Ganin \& Lempitsky, 2015). The goal here is modify the representation $g_{H}(H)$ so that it is maximally informative for NLI while simultaneously minimizes the ability of $c_{\text {Hypoth }}$ to accurately predict the NLI label.

\subsection{AdvDat: Adversarial Training Data}

For our second approach, which we call AdvDat, we use an unchanged general model, but train it with perturbed training data. For a fraction of example $(P, H)$ pairs in the training data, we replace $P$ with $P^{\prime}$, a premise from another training example, chosen uniformly at random. For these instances, during back-propagation, we similarly reverse the gradient but only backpropagate through $g_{H}$. The adversarial loss function $L_{\text {RandAdv }}$ is defined as:

$L\left(c_{\mathrm{NLI}}\left(\left[\operatorname{GRL}_{0}\left(g_{P}\left(P^{\prime}\right)\right) ; \lambda_{\mathrm{Enc}} \operatorname{GRL}_{\lambda}\left(g_{H}(H)\right)\right], y\right)\right)$

where $\mathrm{GRL}_{0}$ implements gradient blocking on $g_{P}$ by using the identity function in the forward step and a zero gradient during the backward step. At the same time, $\mathrm{GRL}_{\lambda}$ reverses the gradient going into $g_{H}$ and scales it by $\lambda_{\text {Enc }}$, as before.

We set a hyper-parameter $\lambda_{\text {Rand }} \in[0,1]$ that controls what fraction $P$ 's are swapped at random. In turn, the final loss function combines the two losses based on $\lambda_{\text {Rand }}$ as

$$
L=\left(1-\lambda_{\text {Rand }}\right) L_{\mathrm{NLI}}+\lambda_{\text {Rand }} L_{\text {RandAdv }}
$$

In essence, this method penalizes the model for correctly predicting $y$ in perturbed examples where the premise is uninformative. This implicitly assumes that the label for $(P, H)$ should be different than the label for $\left(P^{\prime}, H\right)$, which in practice does not always hold true. ${ }^{1}$

\section{Experiments \& Results}

Experimental setup Out of 10 NLI datasets, Poliak et al. (2018) found that the Stanford Natural Language Inference dataset (SNLI; Bowman et al., 2015) contained the most (or worst) hypothesisonly biases - their hypothesis-only model outperformed the majority baseline by roughly $100 \%$ (going from roughly $34 \%$ to $69 \%$ ). Because of the large magnitude of these biases, confirmed

\footnotetext{
${ }^{1}$ As pointed out by a reviewer, a pair labeled as neutral might end up remaining neutral after randomly sampling the premise, so adversarially training in this case might weaken the model. Instead, one could limit adversarial training to cases of entailment or contradiction.
} 
by Tsuchiya (2018) and Gururangan et al. (2018), we focus on SNLI. We use the standard SNLI split and report validation and test results. We also test on SNLI-hard, a subset of SNLI that Gururangan et al. (2018) filtered such that it may not contain unwanted artifacts.

We apply both adversarial techniques to InferSent (Conneau et al., 2017), which serves as our general NLI architecture. ${ }^{2}$ Following the standard training details used in InferSent, we encode premises and hypotheses separately using bi-directional long short-term memory (BiLSTM) networks (Hochreiter \& Schmidhuber, 1997). Premises and hypotheses are initially mapped (token-by-token) to Glove (Pennington et al., 2014) representations. We use max-pooling over the BiLSTM states to extract premise and hypothesis representations and, following Mou et al. (2016), combine the representations by concatenating their vectors, their difference, and their multiplication (element-wise).

We use the default training hyper-parameters in the released InferSent codebase. ${ }^{3}$ These include setting the initial learning rate to 0.1 and the decay rate to 0.99 , using SGD optimization and dividing the learning rate by 5 at every epoch when the accuracy deceases on the validation set. The default settings also include stopping training either when the learning rate drops below $10^{-5}$ or after 20 epochs. In both adversarial settings, the hyper-parameters are swept through $\{0.05,0.1,0.2,0.4,0.8,1.0\}$.

Results Table 2 reports the results on SNLI, with the configurations that performed best on the validation set for each of the adversarial methods.

\begin{tabular}{lrrr}
\hline Model & Val & Test & Hard \\
\hline Baseline & 84.25 & 84.22 & 68.02 \\
\hline AdvCls & 84.58 & 83.56 & 66.27 \\
AdvDat & 78.45 & 78.30 & 55.60 \\
\hline
\end{tabular}

Table 2: Accuracies for the approaches. Baseline refers to the unmodified, non-adversarial InferSent.

As expected, both training methods perform worse than our unmodified, non-adversarial InferSent baseline on SNLI's test set, since they remove biases that may be useful for performing this

\footnotetext{
${ }^{2}$ Code developed is available at https: / / github.com/azpoliak/robust-nli.

${ }^{3}$ https://github.com/facebookresearch/ Infersent
}

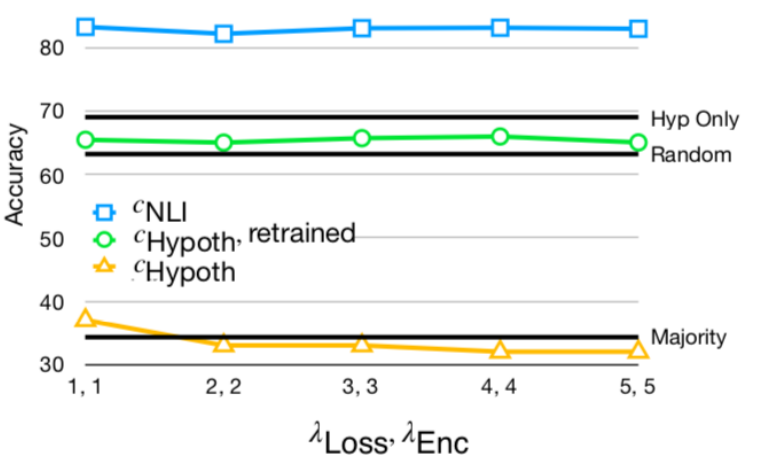

(a) Hidden biases remaining from AdvCls

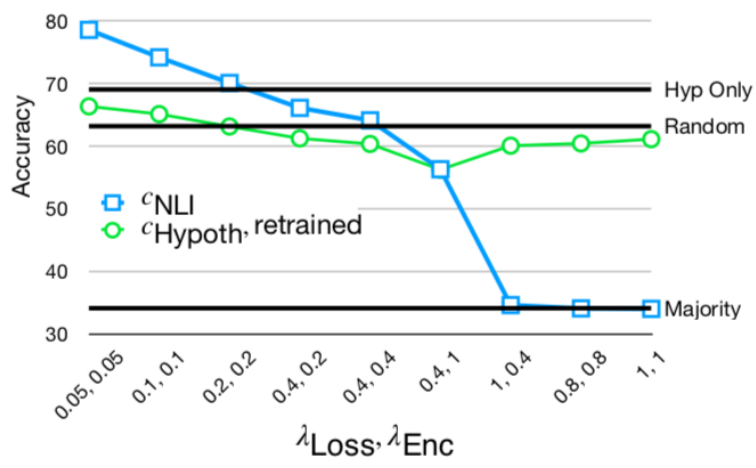

(b) Hidden biases remaining from AdvDat

Figure 1: Validation results when retraining a classifier on a frozen hypothesis encoder ( $c_{\text {Hypoth }}$, retrained) compared to our methods $\left(c_{\mathrm{NLI}}\right)$, the adversarial hypothesisonly classifier ( $c_{\text {Hypoth }}$, in AdvCls), majority baseline, a random frozen encoder, and a hypothesis-only model.

task. The difference for AdvCls is minimal, and it even slightly outperforms InferSent on the validation set. While AdvDat's results are noticeably lower than the non-adversarial InferSent, the drops are still less than $6 \%$ points. $^{4}$

\section{Analysis}

Our goal is to determine whether adversarial learning can help build NLI models without hypothesisonly biases. We first ask whether the models' learned sentence representations can be used by a hypothesis-only classifier to perform well. We then explore the effects of increasing the adversarial strength, and end with a discussion of indicator words associated with hypothesis-only biases.

\subsection{Hidden Biases}

Do the learned sentence representations eliminate hypothesis-only biases after adversarial training?

\footnotetext{
${ }^{4}$ This drop may indicate that SNLI-hard may still have biases, but, as pointed out by a reviewer, an alternative explanation is a general loss of information in the encoded hypothesis. However, Subsection 4.3 suggests that the loss of information is more focused on biases.
} 


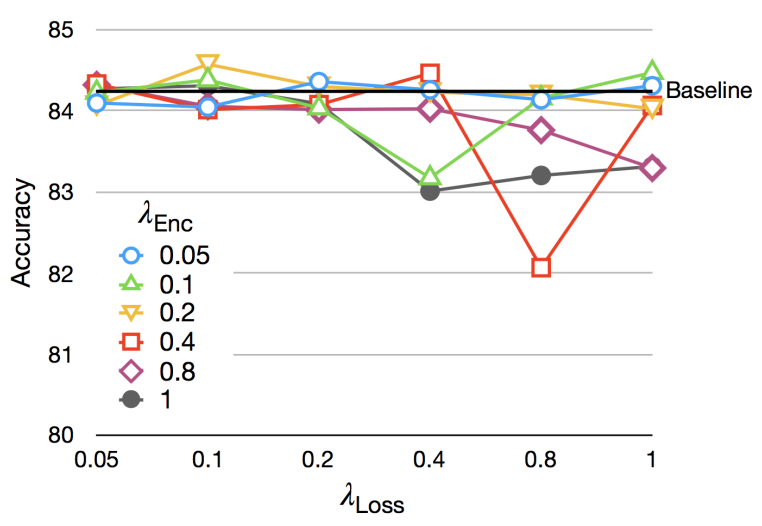

(a) $\mathrm{AdvCls}$

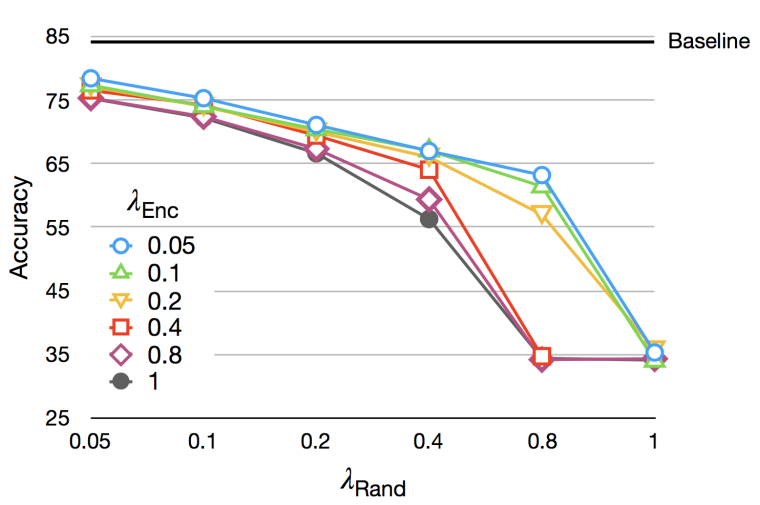

(b) AdvDat

Figure 2: Results on the validation set with different configurations of the adversarial methods.

We freeze sentence encoders trained with the studied methods, and retrain a new classifier that only accesses representations from the frozen hypothesis encoder. This helps us determine whether the (frozen) representations have hidden biases.

A few trends can be noticed. First, we confirm that with AdvCls (Figure 1a), the hypothesisonly classifier $\left(c_{\text {hypoth }}\right)$ is indeed trained to perform poorly on the task, while the normal NLI classifier $\left(c_{\mathrm{NLI}}\right)$ performs much better. However, retraining a classifier on frozen hypothesis representations $\left(c_{\text {Hypoth }}\right.$, retrained) boosts performance. In fact, the retrained classifier performs close to the fully trained hypothesis-only baseline, indicating the hypothesis representations still contain biases. Consistent with this finding, Elazar \& Goldberg (2018) found that adversarially-trained text classifiers preserve demographic attributes in hidden representations despite efforts to remove them.

Interestingly, we found that even a frozen random encoder captures biases in the hypothesis, as a classifier trained on it performs fairly well $(63.26 \%)$, and far above the majority class baseline $(34.28 \%)$. One reason might be that the word embeddings (which are pre-trained) alone contain significant information that propagates even through a random encoder. Others have also found that random encodings contain non-trivial information (Conneau et al., 2018; Zhang \& Bowman, 2018). The fact that the word embeddings were not updated during (adversarial) training could account for the ability to recover performance at the level of the classifier trained on a random encoder. This may indicate that future adversarial efforts should be applied to the word embeddings as well.

Turning to AdvDat, (Figure 1b), as the hyperparameters increase, the models exhibit fewer bi- ases. Performance even drops below the random encoder results, indicating it may be better at ignoring biases in the hypothesis. However, this comes at the cost of reduced NLI performance.

\subsection{Adversarial Strength}

Is there a correlation between adversarial strength and drops in SNLI performance? Does increasing adversarial hyper-parameters affect the decrease in results on SNLI?

Figure 2 shows the validation results with various configurations of adversarial hyperparameters. The AdvCls method is fairly stable across configurations, although combinations of large $\lambda_{\text {Loss }}$ and $\lambda_{\text {Enc }}$ hurt the performance on SNLI a bit more (Figure 2a). Nevertheless, all the drops are moderate. Increasing the hyper-parameters further (up to values of 5), did not lead to substantial drops, although the results are slightly less stable across configurations (Appendix A). On the other hand, the AdvDat method is very sensitive to large hyper-parameters (Figure 2b). For every value of $\lambda_{\text {Enc }}$, increasing $\lambda_{\text {Rand }}$ leads to significant performance drops. These drops happen sooner for larger $\lambda_{\text {Enc }}$ values. Therefore, the effect of stronger hyper-parameters on SNLI performance seems to be specific to each adversarial method.

\subsection{Indicator Words}

Certain words in SNLI are more correlated with specific entailment labels than others, e.g., negation words ("not", "nobody", "no") correlated with CONTRADICTION (Gururangan et al., 2018; Poliak et al., 2018). These words have been referred to as "give-away" words (Poliak et al., 2018). Do the adversarial methods encourage models to make predictions that are less affected by these biased indicator words? 


\begin{tabular}{lrrrrrrr}
\hline & & \multicolumn{2}{c}{ Score } & & \multicolumn{3}{c}{ Percentage decrease from baseline } \\
\cline { 3 - 4 } \cline { 6 - 8 } Word & Count & $\hat{p}(l \mid w)$ & Baseline & & AdvCls $(1,1)$ & AdvDat $(0.4,1)$ & AdvDat $(1,1)$ \\
\hline sleeping & 108 & 0.88 & 0.24 & & 15.63 & 53.13 & -81.25 \\
driving & 53 & 0.81 & 0.32 & & -8.33 & 50 & -66.67 \\
Nobody & 52 & 1 & 0.42 & & 14.29 & 42.86 & 14.29 \\
alone & 50 & 0.9 & 0.32 & & 0 & 83.33 & 0 \\
cat & 49 & 0.84 & 0.31 & & 7.14 & 57.14 & -85.71 \\
asleep & 43 & 0.91 & 0.39 & & -18.75 & 50 & 12.5 \\
no & 31 & 0.84 & 0.36 & & 0 & 52.94 & -52.94 \\
empty & 28 & 0.93 & 0.3 & & -16.67 & 83.33 & -16.67 \\
eats & 24 & 0.83 & 0.3 & & 37.5 & 87.5 & -25 \\
naked & 20 & 0.95 & 0.46 & & 0 & 83.33 & -33.33 \\
\hline
\end{tabular}

Table 3: Indicator words and how correlated they are with CONTRADICTION predictions. The parentheses indicate hyper-parameter values: $\left(\lambda_{\text {Loss }}, \lambda_{\text {Enc }}\right)$ for AdvCls and $\left(\lambda_{\text {Rand }}, \lambda_{\text {Enc }}\right)$ for AdvDat. Baseline refers to the unmodified InferSent.

For each of the most biased words in SNLI associated with the CONTRADICTION label, we computed the probability that a model predicts an example as a contradiction, given that the hypothesis contains the word. Table 3 shows the top 10 examples in the training set. For each word $w$, we give its frequency in SNLI, its empirical correlation with the label and with InferSent's prediction, and the percentage decrease in correlations with CONTRADICTION predictions by three configurations of our methods. Generally, the baseline correlations are more uniform than the empirical ones $(\hat{\mathrm{p}}(l \mid w))$, suggesting that indicator words in SNLI might not greatly affect a NLI model, a possibility that both Poliak et al. (2018) and Gururangan et al. (2018) do concede. For example, Gururangan et al. (2018) explicitly mention that "it is important to note that even the most discriminative words are not very frequent."

However, we still observed small skews towards CONTRADICTION. Thus, we investigate whether our methods reduce the probability of predicting CONTRADICTION when a hypothesis contains an indicator word. The model trained with AdvDat (where $\lambda_{\text {Rand }}=0.4, \lambda_{\text {Enc }}=1$ ) predicts contradiction much less frequently than InferSent on examples with these words. This configuration was the strongest AdvDat model that still performed reasonably well on SNLI (Figure 2b). Here, AdvDat appears to remove some of the biases learned by the baseline, unmodified InferSent. We also provide two other configurations that do not show such an effect, illustrating that this behavior highly depends on the hyper-parameters.

\section{Conclusion}

We employed two adversarial learning techniques to a general NLI model by adding an external adversarial hypothesis-only classifier and perturbing training examples. Our experiments and analyses suggest that these techniques may help models exhibit fewer hypothesis-only biases. We hope this work will encourage the development and analysis of models that include components that ignore hypothesis-only biases, as well as similar biases discovered in other natural language understanding tasks (Schwartz et al., 2017), including visual question answering, where recent work has considered similar adversarial techniques for removing language biases (Ramakrishnan et al., 2018; Grand \& Belinkov, 2019).

\section{Acknowledgements}

This work was supported by JHU-HLTCOE, DARPA LORELEI, and the Harvard Mind, Brain, and Behavior Initiative. We thank the anonymous reviewers for their comments. Views and conclusions contained in this publication are those of the authors and should not be interpreted as representing official policies or endorsements of DARPA or the U.S. Government.

\section{References}

Moustafa Alzantot, Yash Sharma, Ahmed Elgohary, Bo-Jhang Ho, Mani Srivastava, and Kai-Wei Chang. Generating Natural Language Adversarial Examples. In Proceedings of the 2018 Conference on 
Empirical Methods in Natural Language Processing, pp. 2890-2896, 2018.

Yonatan Belinkov and Yonatan Bisk. Synthetic and Natural Noise Both Break Neural Machine Translation. In International Conference on Learning Representations, 2018. URL https:// openreview.net/ forum?id=BJ8vJebC-.

Samuel R. Bowman, Gabor Angeli, Christopher Potts, and Christopher D. Manning. A large annotated corpus for learning natural language inference. In Proceedings of the 2015 Conference on Empirical Methods in Natural Language Processing (EMNLP). Association for Computational Linguistics, 2015.

Xilun Chen and Claire Cardie. Multinomial adversarial networks for multi-domain text classification. In Proceedings of the 2018 Conference of the North American Chapter of the Association for Computational Linguistics: Human Language Technologies, Volume 1 (Long Papers), pp. 1226-1240. Association for Computational Linguistics, 2018. URL http://aclweb.org/ anthology/N18-1111.

Alexis Conneau, Douwe Kiela, Holger Schwenk, Loïc Barrault, and Antoine Bordes. Supervised Learning of Universal Sentence Representations from Natural Language Inference Data. In Proceedings of the 2017 Conference on Empirical Methods in Natural Language Processing, pp. 670-680. Association for Computational Linguistics, 2017. URL http : / / aclweb.org/anthology/D17-1070.

Alexis Conneau, Germán Kruszewski, Guillaume Lample, Loïc Barrault, and Marco Baroni. What you can cram into a single $\$ \&$ !\#* vector: Probing sentence embeddings for linguistic properties. In Proceedings of the 56th Annual Meeting of the Association for Computational Linguistics (Volume 1: Long Papers), pp. 2126-2136. Association for Computational Linguistics, 2018. URL http: // aclweb.org/anthology/P18-1198.

Yanai Elazar and Yoav Goldberg. Adversarial Removal of Demographic Attributes from Text Data. In Proceedings of the 2018 Conference on Empirical Methods in Natural Language Processing, pp. 11-21. Association for Computational Linguistics, 2018. URL http://aclweb.org/ anthology/D18-1002.

Yaroslav Ganin and Victor Lempitsky. Unsupervised Domain Adaptation by Backpropagation. In International Conference on Machine Learning, pp. 1180-1189, 2015.

Ian J Goodfellow, Jonathon Shlens, and Christian Szegedy. Explaining and Harnessing Adversarial Examples. In International Conference on Learning Representations (ICLR), 2015.

Gabriel Grand and Yonatan Belinkov. Adversarial Regularization for Visual Question Answering:
Strengths, Shortcomings, and Side Effects. In Proceedings of the 2nd Workshop on Shortcomings in Vision and Language (SiVL) at NAACL-HLT, June 2019.

Suchin Gururangan, Swabha Swayamdipta, Omer Levy, Roy Schwartz, Samuel Bowman, and Noah A. Smith. Annotation Artifacts in Natural Language Inference Data. In Proceedings of the 2018 Conference of the North American Chapter of the Association for Computational Linguistics: Human Language Technologies, Volume 2 (Short Papers), pp. 107-112, New Orleans, Louisiana, June 2018. Association for Computational Linguistics. URL http: //www.aclweb.org/anthology/N18-2017.

Sepp Hochreiter and Jürgen Schmidhuber. Long shortterm memory. Neural computation, 9(8):17351780, 1997.

Dongyeop Kang, Tushar Khot, Ashish Sabharwal, and Eduard Hovy. AdvEntuRe: Adversarial Training for Textual Entailment with Knowledge-Guided Examples. In Proceedings of the 56th Annual Meeting of the Association for Computational Linguistics (Volume 1: Long Papers), pp. 2418-2428, Melbourne, Australia, July 2018. Association for Computational Linguistics. URL http: / / www.aclweb.org/ anthology/P18-1225.

Pasquale Minervini and Sebastian Riedel. Adversarially Regularising Neural NLI Models to Integrate Logical Background Knowledge. In Proceedings of the 22nd Conference on Computational Natural Language Learning (CoNLL 2018). Association for Computational Linguistics, 2018.

Lili Mou, Rui Men, Ge Li, Yan Xu, Lu Zhang, Rui Yan, and Zhi Jin. Natural Language Inference by Tree-Based Convolution and Heuristic Matching. In Proceedings of the 54th Annual Meeting of the Association for Computational Linguistics (Volume 2: Short Papers), pp. 130-136, Berlin, Germany, August 2016. Association for Computational Linguistics. URL http: / / anthology.aclweb.org/ P16-2022.

Jeffrey Pennington, Richard Socher, and Christopher Manning. Glove: Global vectors for word representation. In Proceedings of the 2014 conference on empirical methods in natural language processing (EMNLP), pp. 1532-1543, 2014.

Adam Poliak, Jason Naradowsky, Aparajita Haldar, Rachel Rudinger, and Benjamin Van Durme. Hypothesis Only Baselines in Natural Language Inference. In Proceedings of the Seventh Joint Conference on Lexical and Computational Semantics, pp. 180-191, New Orleans, Louisiana, June 2018. Association for Computational Linguistics. URL http : //www.aclweb.org/anthology/s18-2023.

Sainandan Ramakrishnan, Aishwarya Agrawal, and Stefan Lee. Overcoming Language Priors in Visual Question Answering with Adversarial Regularization. In NIPS, pp. 1548-1558, 2018. 
Roy Schwartz, Maarten Sap, Ioannis Konstas, Leila Zilles, Yejin Choi, and Noah A. Smith. Story Cloze Task: UW NLP System. In Proceedings of LSDSem, 2017.

Masatoshi Tsuchiya. Performance Impact Caused by Hidden Bias of Training Data for Recognizing Textual Entailment. In 11th International Conference on Language Resources and Evaluation (LREC2018), 2018.

Qizhe Xie, Zihang Dai, Yulun Du, Eduard Hovy, and Graham Neubig. Controllable invariance through adversarial feature learning. In Advances in Neural Information Processing Systems, pp. 585-596, 2017.

Brian $\mathrm{Hu}$ Zhang, Blake Lemoine, and Margaret Mitchell. Mitigating unwanted biases with adversarial learning. In Proceedings of the 2018 AAAI/ACM Conference on AI, Ethics, and Society, pp. 335-340. ACM, 2018.

Kelly Zhang and Samuel Bowman. Language Modeling Teaches You More than Translation Does: Lessons Learned Through Auxiliary Task Analysis. In Proceedings of the 2018 EMNLP Workshop BlackboxNLP: Analyzing and Interpreting Neural Networks for NLP, pp. 359-361, Brussels, Belgium, November 2018. Association for Computational Linguistics. URL http://www.aclweb.org/ anthology/W18-5448.

\section{A Stronger hyper-parameters for AdvCls}

Figure 3 provides validation results using $\mathrm{AdvCls}$ with stronger hyper-parameters to complement the discussion in $\S 4.2$. While it is difficult to notice trends, all configurations perform similarly and slightly below the baseline. These models seem to be less stable compared to using smaller hyperparameters, as discussed in $\S 4.2$.

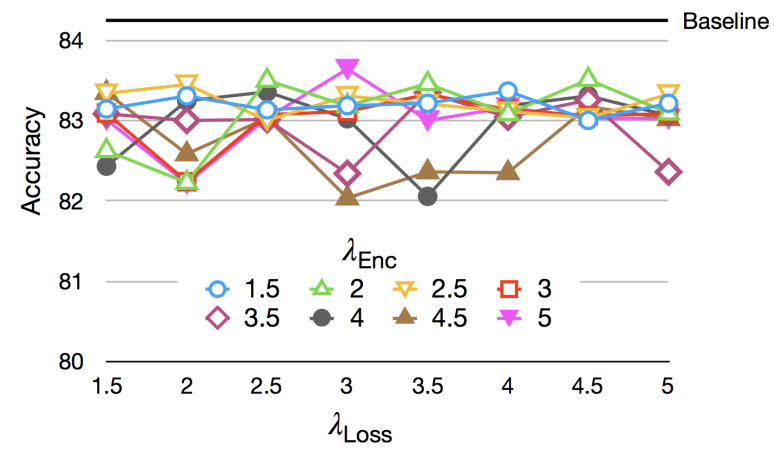

Figure 3: Validation results using AdvCls with stronger hyper-parameters. 